\title{
La spécificité de l'enseignement de la biologie : entre le repli défensif et l'ouverture
}

\section{Guy Rumelhard}

\section{Q OpenEdition}

1 Journals

\section{Édition électronique}

URL : http://journals.openedition.org/trema/1987

DOI : 10.4000/trema.1987

ISSN : 2107-0997

\section{Éditeur}

Faculté d'Éducation de l'université de Montpellier

\section{Édition imprimée}

Date de publication : 1 septembre 1996

Pagination : 23-32

ISSN : 1167-315X

\section{Référence électronique}

Guy Rumelhard, «La spécificité de l'enseignement de la biologie : entre le repli défensif et l'ouverture », Tréma [En ligne], 9-10 | 1996, mis en ligne le 11 septembre 2013, consulté le 01 mai 2019. URL : http:// journals.openedition.org/trema/1987 ; DOI : 10.4000/trema.1987

Ce document a été généré automatiquement le 1 mai 2019.

Trema 


\title{
La spécificité de l'enseignement de la biologie : entre le repli défensif et l'ouverture
}

\author{
Guy Rumelhard
}

1 S'interroger sur la spécificité de la Biologie et de son enseignement peut sembler a priori quelque peu « défensif ». Sommes-nous si mal assurés de notre objet d'étude ? Conteste-ton que la Biologie soit «la science de la vie», comme d'autres auteurs contestent la Psychologie comme science de l'esprit? Sommes-nous mal assurés de notre scientificité? La Biologie n'est-elle pas déterministe et expérimentale ? Serait-elle toujours considérée comme une " histoire de la nature », une sorte de grande fresque des êtres vivants tant au niveau de la recherche que de l'enseignement? Mais que signifie exactement interdisciplinarité ? Au niveau de la recherche la spécialisation semble indispensable, même si les objets nouveaux sont construits par la collaboration de plusieurs disciplines. Au niveau de l'enseignement il s'agit plutôt d'un mot d'ordre polémique désignant positivement le fait supposé que la valeur culturelle et éducative d'une matière réside dans la transversalité de ses concepts et des méthodes. Négativement le mot désigne une position étroite, limitée, bornée, ou réservée à un petit nombre, et n'ayant pas de valeur éducative "générale». La valeur principale d'un enseignement scientifique résiderait dans son initiation à la méthode scientifique, mieux, à la démarche scientifique, mieux encore à l'esprit scientifique. C'est donc sa finalité comme matière d'enseignement qui doit être interrogée, discutée et « défendue ». 
Louis Pasteur, (1822-1895).

Tableau de Robert Thom. Collection du Musée Pasteur.

Les Cahiers de la Science, Paris, Excelsior Publ., N4, août 1991.

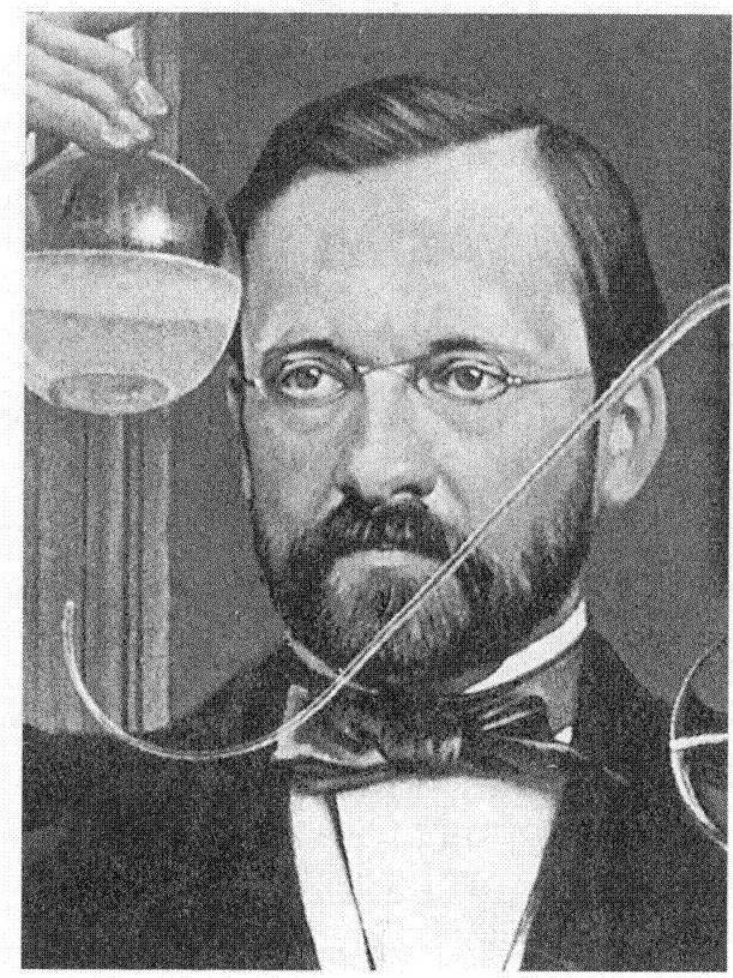

\section{Instruments et expériences : un rationalisme de laboratoire}

2 Contre une médecine empirique qui bien souvent se contente de nommer, décrire et classer, Claude Bernard, en 1865 ouvre le chemin d'un idéal déterministe et popularise cette idée sous le vocable de "médecine expérimentale ». Depuis, l'habitude a souvent été prise de regrouper certaines sciences physiques, chimiques, biologiques sous la bannière commune de "sciences expérimentales ». Ici encore l'heure est au rassemblement plutôt qu'à la recherche d'une spécificité, même s'il apparaît rapidement que l'astronomie, la géologie, la théorie de l'évolution, la méthode pathologique dans laquelle les maladies sont considérées comme des expériences, la méthode comparative, etc., ne sont pas " expérimentales». Dans l'enseignement les vocables de sciences expérimentales", " enseignement scientifique ", " enseignement expérimental » recouvrent bien souvent diverses sciences et même parfois des techniques. Faut-il y voir l'effet des attaques administratives (un professeur de « sciences » enseigne plusieurs matières), ou d'attaques pédagogiques contre la notion de matière au nom d'objectifs réputés «supérieurs » car plus transversaux?

3 Jean Rostand, qui dirige en 1965 le volume Biologie dans l'Encyclopédie de la Pléiade, détaille plusieurs aspects de cette "défense et illustration» de la Biologie dans son introduction. Tout d'abord ce que l'on pourrait nommer avec Gaston Bachelard, un " engagement rationaliste ", la nécessité d'une attitude militante pour continuer à justifier et promouvoir le fait que la Biologie et la Médecine sont de véritables sciences car elles 
s'appuient sur une méthode et des expériences de laboratoire. Cent ans après Claude Bernard et sa célèbre "Introduction à l'étude de la médecine expérimentale» la conviction reste encore à renforcer.

4 Mais précisément les deux figures toujours bien présentes de Pasteur et de Claude Bernard, mal lues et déformées, conduisent à privilégier, dans le travail scientifique, le travail de laboratoire, l'utilisation d'instruments, de techniques ainsi que l'observation. Le savoir est avéré par les faits. Bien évidemment ce travail ne se limite plus à la simple exploration d'un donné. Il ne se limite plus à nommer, décrire et classer. Le biologiste est inventeur de phénomènes, un contremaître de la création qui produit une nature nouvelle, qui ajoute à la réalité grâce à des artifices expérimentaux. Le médecin se méfie des descriptions de symptômes, et des classifications. Il explore des fonctions. Mais l'essentiel se déroule au laboratoire ou dans les cliniques.

Photo de Claude Bernard, (1813-1878).

Savant, contemporain de Pasteur, et membre du Collège de France.

Les Cahiers de la Science, Paris, Excelsior Publ., Nº, août 1991.

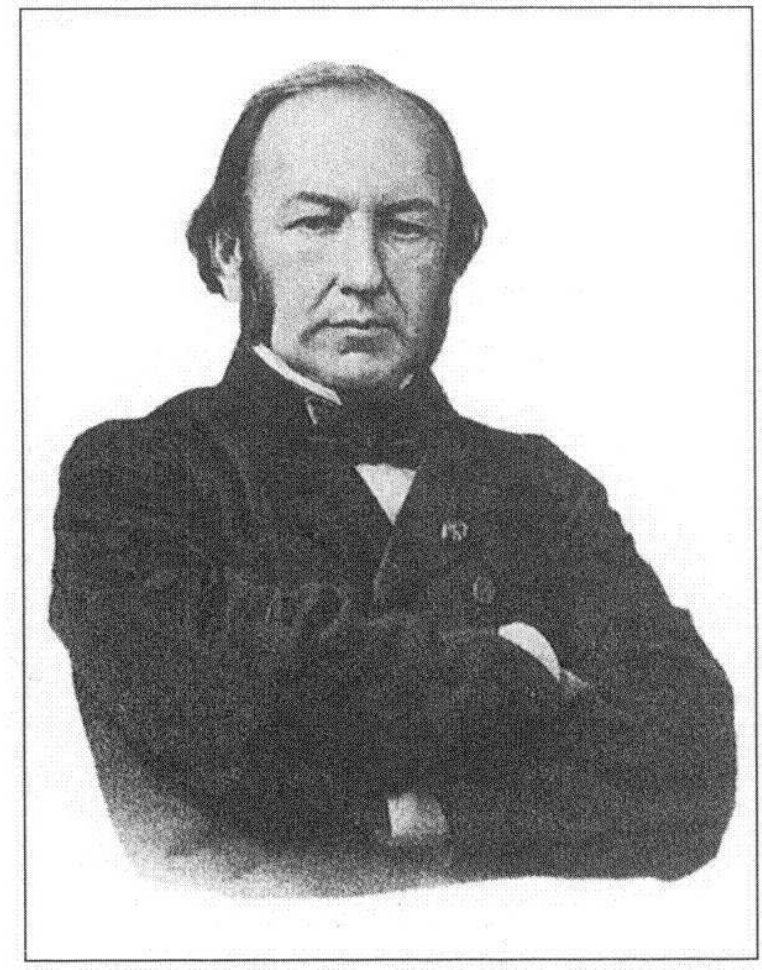

Ce rationalisme de laboratoire se double d'une injonction matérialiste et antimétaphysique : expliquer la vie sans la vie, en ne faisant appel qu'à la physico-chimie ou aux mathématiques. Si les sciences physico-chimiques ont, en quelque sorte, "dématérialisé " la matière en exprimant certaines "particules» en tenues mathématiques et probabilistes, les biologistes se fixent comme idéal de «dévitaliser » la vie. La vie n'existe pas, c'est un concept métaphysique, et il faut bien évidemment exorciser toute tentation métaphysique non pas à la marge comme pouvait l'être l'alchimie pour la chimie, mais bien au cour du savoir.

6 Cette vigilance anti-métaphysique doit également s'exercer vis-à-vis de toute intrusion idéologique. En 1965 l'affaire Lyssenko vient juste de s'achever. Cette ingérence de l'idéologie dans la science est allée jusqu'à faire dire qu'il existe une biologie bourgeoise 
et une biologie prolétarienne. Mais cette "politisation" n'est ni la première, ni la dernière. L'eugénisme, le racisme, sont venus et viennent encore chercher des arguments dans la biologie depuis 1860 .

7 Sur le plan de la recherche, l'attitude «mécaniste » est un réductionnisme de méthode qui s'est avéré seul fécond et efficace. Le vitalisme n'a produit aucun concept réellement opératoire. Mais cette attitude de principe se convertit aisément en un antiphilosophisme à cause des deux dernières raisons évoquées. Il plane sur l'enseignement de la biologie une méfiance vis-à-vis de toute spéculation théorique qui conduit à séparer nettement les hypothèses des faits, qui conduit à considérer les faits comme des acquis solides et les interprétations comme fragiles et soumises à rectification permanente.

Claude Bernard fixe un idéal déterministe à la médecine, mais c'est Pasteur qui apporte les premières actions concrètes qui permettent de soigner réellement et surtout de prévenir. Depuis cette époque biologie et médecine sont et doivent apparaître comme étroitement liées. La physiologie vient chercher au chevet des malades ses questions et ses problèmes, la médecine vient chercher au laboratoire des gestes et des médicaments nouveaux. Le chemin est court, et l'urgence demande impérativement que le délai de cheminement soit bref. Tout détour théorique peut sembler une perte de temps. La spéculation doit devenir rapidement opératoire. Toute recherche a nécessairement des applications médicales, ou du moins il faut en convaincre ceux qui financent. Et si l'on peut concevoir une "biologie théorique » qui prend naissance il y a une vingtaine d'années, sans entrer dans l'enseignement, on ne conçoit pas l'existence d'une " médecine théorique ».

\section{Pratique, pragmatisme, activité, opératoire}

Un courant pédagogique presque unanimement invoqué à travers le mot d'ordre de méthode activerecouvre en fait des intentions et des attitudes variées, dont certaines viennent converger avec cette méfiance vis-à-vis de toute spéculation. John Dewey, pédagogue et cofondateur d'une philosophie typiquement américaine : le pragmatisme, privilégie l'activité pratique des élèves et la résolution de problèmes pratiques. Cette pédagogie du "problem solving» vient en lutte contre un enseignement uniquement rhétorique et verbal. Elle est censée fournir un moteur à l'apprentissage et un intérêt qui facilite l'assimilation et la rend plus facile. Le savoir appris prend un caractère «fonctionnel » puisqu'il répond à une question posée, question qui est ou qui devient celle de l'élève. Le côté "exercice artificiel ", exercice préparatoire ou introductif à un « vrai savoir » disparait.

Selon Charles S. Peirce l'esprit du pragmatisme c'est l'esprit expérimentaliste ou l'esprit de laboratoire. Le pragmatisme est une philosophie de la méthode scientifique : c'est un expérimentalisme. Ce n'est pas une philosophie des résultats, ce n'est pas un scientisme. L'idée est immédiatement une hypothèse et un plan d'action, sinon elle n'a pas de sens. Mais il n'y a pas lieu d'opposer l'idée d'un savant et celle de l'homme de la rue. Toutes deux doivent être expérimentées. De même il n'y a pas d'idées définitivement établies. La mise à l'épreuve est toujours une éventuelle remise en question. D'une certaine façon, le pragmatisme ne tente pas de déterminer la vérité des choses. Ce n'est toutefois pas un simple "practicisme » ou "practicalisme » selon les expressions que W. James aurait voulu imposer, car il recherche cependant la signification des concepts abstraits. Ce n'est pas un anti-intellectualisme, mais c'est pourtant sous cet aspect que le mot s'est répandu en 
Europe et a été repris par plusieurs courants pédagogiques dans des perspectives diverses chez O. Decroly ou C. Freinet.

11 C. Freinet se souciait peu de théoriser sa pratique, et les enseignants pratiquentvolontiers le « grappillage » sans souci de cohérence théorique ou de théorie de l'apprentissage. Ils font feu de tout bois concret, de tout procédé, de toute activité proposée pourvu qu'elle réussisse. Le rationalisme de laboratoire précédemment décrit converge donc avec l'injonction des méthodes actives, des activités pratiques dans une méfiance vis-à-vis de toute spéculation intellectuelle. Il est peu utile de souligner à quel point le terme de pragmatique est valorisé dans le vocabulaire politique en s'opposant violemment à toute attitude doctrinale, idéologique ou simplement spéculative.

Photo de Célestin Freinet, (1896-1966).

Freinet parmi ses élèves, à Saint Paul de Vence, vers 1930.

GAULUPEAU Y., La France à l'Ecole, Paris, Gallimard, 1992, Ré-éd. : 1994.

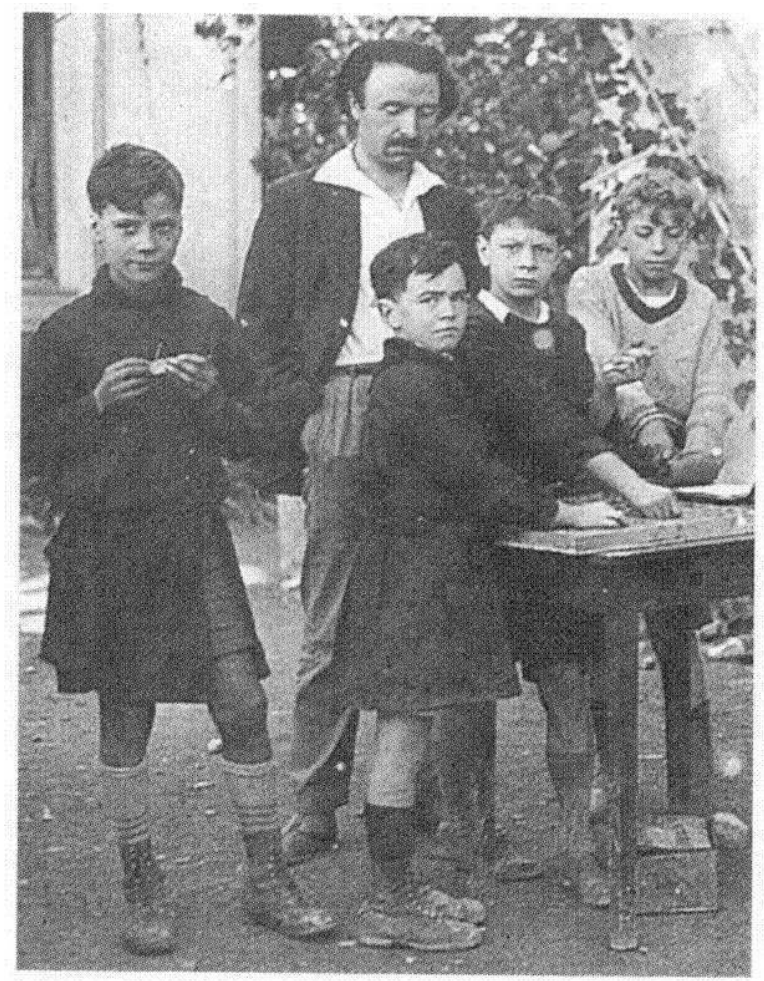

12 Chez Freinet sa conception pédagogique se colore d'une philosophie du travail à consonance politique. Elle converge avec une réflexion plus épistémologique sur le rôle de la technique, et de la pratique. Althusser étendra ce concept de pratique non seulement à l'activité technique, mais également aux représentations, aux institutions, à la pratique théorique».

Piaget mettra de son côté l'accent sur une conception active et opérative de la connaissance. Il popularise la formule «comprendre c'est opérer ». Cet aphorisme se traduit concrètement par des travaux de psychologie expérimentale visant à constituer une théorie de la connaissance sur une base qui puisse trouver un accord dans les divers pays. Dans ce but il pense indispensable de reconduire à la frontière toute philosophie.

14 La philosophie est renvoyée du côté de la littérature, et une nouvelle fois le mythe du divorce émancipateur de la science (y compris la science de l'enfant) d'avec la philosophie 
conduit à survaloriser l'expérimentalisme. La méthode expérimentale (souvent écrite au singulier) devient en elle-même un critère de scientificité et une protection contre toute intrusion des idéologies, des mythes, des représentations, des idéologies.

\section{Le repli derrière la frontière}

Ce couplage très fort entre ACTIVITÉ, MÉTHODE, TRAVAIL, PRATIQUE, OPÉRATIVITÉ, EXPÉRIMENTATION semble définir une position solide. L'objet d'étude se présentant avec l'évidence d'un donné (la vie, les vivants, les maladies), l'utilité pratique de ce savoir dans le domaine de l'alimentation, de la santé, de l'environnement n'étant pas contesté, le caractère motivant de ces sujets d'étude n'appelant pas de réserves, les enseignants comprennent mal les attaques qui contestent son rôle comme discipline d'enseignement à valeur éducative.

\subsection{Faire et découvrir}

L'épistémologie administrative admet deux pôles de l'éducation scientifique : le fait et le calcul. La Biologie est donc rangée du côté de la science des faits d'observation. Le savoir est avéré par les faits et ceci est accessible dès le jeune âge. Mais son risque permanent est l'inflation de la mémoire. Le côté opératoire est presque absent, ou réservé à quelques chapitres tels la Génétique. Les modèles utilisés sont essentiellement analogiques. Et Piaget a opposé le figuratif et l'opératif. Biologiste de formation initiale, il s'est spécialisé dans la logique (au singulier) entendue comme science universelle du (au singulier) raisonnement scientifique. En Biologie, cette logique semble se limiter à la logique des classes. Sinon il s'agit de « découvrir ».

$\mathrm{Du}$ côté administratif on peut bien évidemment ajouter des arguments négatifs concernant le coût financier de la réalisation en classe de travaux pratiques (matériels, petits effectifs, préparateur, salle spécialisée).

Aux États-Unis d'Amérique du Nord l'enseignement des High-Schools a été fortement influencé par les conceptions de J. Dewey et du pragmatisme. Depuis les premiers textes de Hanna Arendt, traduits tardivement en 1972, sur « la crise de la culture », les critiques n'ont cessé de s'amplifier. Dewey pense que l'activité pratique et la résolution de problèmes donnent un intérêt à l'apprentissage. Cet intérêt constitue le moteur du travail personnel et un facilitateur de l'assimilation. Mais la critique porte sur l'idée suivante: « on ne peut savoir et comprendre que ce que l'on fait ». Et cette idée prise à la lettre a bien souvent conduit à substituer le faire à l'apprendre, sinon au comprendre. Or la distinction entre comprendre et faire est une distinction bien réelle, même si l'on admet qu'on ne comprend vraiment que ce que l'on fait. À moins d'apprendre des gestes par imitation de gestes, il est indispensable de se faire une représentation de l'action. La compréhension donne son sens au faire, elle ouvre de nouvelles possibilités. La compréhension n'est pas un but en soi et le faire donne alors sa sanction au comprendre. Mais il reste que l'on comprend à partir de principes qui sont des jugements, et que l'on fait à partir de procédés qui sont des gestes.

19 L'enseignement aurait fait preuve d'un intellectualisme pur en oubliant que savoir pour savoir n'a pas de sens. L'intellectualisme avait créé un faux conflit entre la connaissance et la vie. Or la signification du détour théorique, le sens de connaître, est bien de vivre 
mieux. Au lieu de poser la question en termes différents, l'enseignement américain a bien souvent seulement renversé les termes de la fausse opposition. Privilégions le faitdevivre au présent, de faire, et d'y trouver du plaisir. Il ne s'agit pas de faire des exercices préparatoires à... Letravailréel (on dit aussi fonctionnel) est en lui même moteur, principe de compréhension, principe de réalité, principe de plaisir, principe d'autonomisation.

\subsection{La signification des connaissances scientifiques}

Si le «faire » ne s'articule pas à un raisonnement et à un jugement porté sur les faits, il devient une "découverte", et l'on connait la très grande fortune de ce mot au niveau publicitaire. Mais la question de la signification du savoir scientifique et de sa valeur éducative ne se limite pas aux relations entre comprendre et faire.

21 Existe-t-il un enseignement scientifique qui ne se pose pas la question de sa valeur éducative, ou bien y répond par une affirmation de suffisance ou de pureté : savoir pour savoir? Disons que, bien souvent, il n'est pas aisé de trouver une réponse dans les textes administratifs qui définissent la nécessité de cet enseignement.

Initialement le pragmatisme est une philosophie de la démocratie. Il s'interroge donc sur la signification du savoir et sur la façon de le relier à l'égalité et à la liberté. Les Encyclopédistes et Condorcet avaient déjà contribué à poser cette question en termes de philosophie politique. Mais il y a plusieurs façons de relier démocratie, liberté, égalité et laïcité. Et ces réponses s'opposent violemment. À l'époque de J. Dewey, Charles W. Eliot, président de l'Université Harvard, se pose également comme propagandiste infatigable et persuasif d'une «éducation démocratique ». Mais pour lui l'idéal d'égalité consiste à permettre à tous les citoyens d'exploiter au mieux leurs différences naturelles. Une sorte « d'élitisme républicain » qui fait écho à un débat d'une étonnante actualité.

L'antiphilosophisme évoqué précédemment a largement contribué à éliminer cette question posée en termes de philosophie politique et à la transposer en une question de morale. Pour les responsables d'une défense corporatiste de la discipline il s'agit de transmettre des connaissances un peu comme on transmet un bien que l'on possède. Le savoir scientifique trouve, à travers ses applications, une valeur morale. Les revendications «d'humanisme scientifique» n'osent plus l'affirmer comme tel. Mais, bien mieux que l'appel à la «formation d'un esprit scientifique » qui croyait trouver une caution chez Gaston Bachelard compris à contre-sens, c'est la médecine qui donne à la biologie sa valeur morale. À mesure que s'aggrave la débacle des valeurs qui réglaient la pensée des sociétés modernes, on voit la santé devenir l'ultime refuge, et même la référence absolue. Le personnage du médecin incarne, presque seul, le dévouement à autrui, le sens du devoir, et à l'occasion l'audace et le goût du risque. La biologie en tire profit puisque la recherche est «biomédicale ». La médecine et la santé sont devenues un corps et une institution sociale avec les concepts de prévention, prédiction, préservation et surveillance. Cette idéologie morale est, en son fond, ancrée dans la peur et la crainte. L'écologie apporte son idéologie de la conservation et l'immunologie celle d'une individualisation et d'un repli sur « le soi ».

Certains didacticiens qui veulent produire un savoir sur « le comment apprendre », et les obstacles à l'assimilation-appropriation, rejettent cette question du côté de la pédagogie ou du pouvoir politique, contribuant ainsi à dissocier la question des procédés d'enseignement de celle des finalités. Revenons une nouvelle fois à Dewey qui rattache quant à lui cette question à la psychologie expérimentale naissante. Le sens des 
connaissances se trouve dans le développement et l'épanouissement de l'enfant. Et cette "science de l'enfant» va nous apporter des réponses appropriées. Mais l'idée d'une psychologie expérimentale qui prétend trouverson autonomie et ses fondements dans sa méthode, inclut sans le dire la fiction métaphysique d'une Nature Humaine. L'enfant trouve en lui même ses propres normes de développement. Toute volonté de lui imposer de l'extérieur des normes éducatives est à rejeter. Cette psychologie souvent généreuse et bien intentionnée enferme en fait une vision « utilitaire » de la personne qui refuse de se dire comme telle et impose en fait d'autres normes de comportement. Prétendre produire un savoir sur l'enfant, ou seulement une méthode d'observation de l'enfant évacue la philosophie politique, mais ne nous fait pas quitter la philosophie morale.

L'essentiel de cette activité d'enseignement scientifique suppose non seulement des faits à observer et mémoriser, des méthodes (au pluriel) à inventer dans chaque situation, mais également des jugements de vérité qui engagent le tout de la pensée humaine, et donc de la vie humaine. L'essentiel de l'enseignement scientifique réside non pas tant dans ses applications utiles ou sa valeur morale, que dans sa valeur émancipatrice toujours à repenser et actualiser. Et ceci est et reste une question de philosophie politique.

\section{Les questions à rouvrir}

Dans ce bref article on se contentera d'énumérer les questions à analyser, ou plus exactement à rouvrir ou réintroduire au cœur de l'enseignement scientifique.

\subsection{Identifier les concepts}

Les concepts comme outils théoriques (concepts de savoirs, de techniques, de méthodes, de causalités). Le terme de concept est d'introduction récente dans le vocabulaire des scientifiques et de l'enseignement. Mais peu importe l'usage d'un terme de plus en plus abondamment utilisé d'ailleurs dans le langage de la publicité. Chacun identifiera dans les mots :

- régulation, hormone, milieu intérieur, enzyme,

- observation, hypothèse, expérience,

- cause, effet, rétroaction, interaction,

- électrophorèse, centrifugation, anticorps monoclonaux,

la désignation de quelque chose qui n'est pas simplement un résultat factuel. On peut dire que certains mots sont des "outils théoriques" car ils permettent de créer des distinctions (couper comme un scalpel), ou rassembler des observations disparates (coudre comme une aiguille), ouvrir un champ de recherche et relancer l'invention (comme un ressort tendu), guider un plan de travail, identifier un type de relation.

Inversement on peut dire que chaque instrument implique beaucoup plus de théorie qu'il n'y parait. Un simple microscope implique d'adhérer à une théorie du pouvoir séparateur, aux mécanismes de réfraction, de diffraction dans les structures fines etc.

Un important travail reste à faire pour analyser des concepts moins classiques: organisation fonctionnelle, distance fonctionnelle, localisation/délocalisation des centres nerveux, des organes du système immunitaire, le normal et le pathologique, le qualitatif et le quantitatif, la place des mathématiques probabilistes comme outils de description, comme méthode d'organisation des expériences, comme modèle explicatif, comme 
explication causale, le continu et le discontinu, la causalité systémique, la méthode pathologique, la méthode toxicologique, la méthode du placébo en double aveugle, etc.

\subsection{Repérer les représentations}

31 L'analyse de représentations comme aide ou obstacle à l'assimilation de savoirs, des techniques, des méthodes expérimentales, des types de causalité, est certainement l'apport le plus novateur et la contribution la plus critique de l'épistémologie à la didactique. Mais il est dans la nature même des représentations d'être niées, recouvertes, raturées, limitées, restreintes, rétrécies à ce qu'elles disent explicitement (d'où le terme ambigu et positif de représentation). Le travail est ici perpétuellement à refaire, à rouvrir, comme une dure épreuve qui se présente au cœur même du savoir et qui est toujours rejetée à la marge.

\subsection{Expliciter les idéologies sous-jacentes}

La vigilance toujours en éveil vis-à-vis des idéologies pensées non pas comme intrusion extérieures et dont on pourrait se protéger à l'aide d'une muraille infranchissable, mais comme des sécrétions au cœur même du travail d'enseignement: de la guérison des maladies à la prévention (vaccins...) et à la prédiction (génétique), il y a un passage non dit ni analysé de l'individu au social, au politique, et l'institution du médical en «référence absolue »; de l'écologie à la protection-conservation de la nature, il faut inclure le concept de responsabilité; les concepts de programme génétique, de surveillance immunitaire, sont-ils aussi scientifiquement neutres qu'il y paraît ? Existe-til une définition scientifique de la santé ou de la physiologie normale?

\subsection{Des savoirs biologiques : pour quel usage social?}

La vigilance vis-à-vis d'un biologisme social, historique, psychologique, politique. La biologie semble apporter comme des données de fait indubitables les concepts de Nature, de Vie, de Santé, de Régulation, d'Unité, d'Unicité, de Développement, d'Adaptation et bien d'autres, censés permettre de penser directement les éléments de la vie affective et sociale (et de l'assimilation du savoir).

\subsection{Introduire l'épistémologie historique}

L'épistémologie historique n'a pas encore produit tous les effets attendus de l'importation de ses concepts en didactique. La critique du schéma stéréotypé de présentation de la méthode expérimentale (observation, hypothèse, expérience) est restée inachevée. De même les relations entre processus de découverte et produit, modélisation conceptualisation, objectivation et objet, entre le construit et les données de fait. De même les procédures de validation, de réfutation, de discussion critique, de jugement de vérité conduisant à l'objectivité du savoir. De même la formulation des problèmes, la dynamique d'évolution de la recherche, le recul des frontières du savoir et la formulation des concepts en attente. De même encore, la capacité d'intégration de l'imprévu, la capacité de réorganisation, de structuration, de changement à la base. De même enfin l'approfondissement de l'éthique scientifique et médicale. 


\subsection{Un réductionnisme nécessaire mais à surveiller} d'explication, qui tend en permanence à se transformer en philosophie biologique et à clore ou du moins limiter ou interdire certaines questions. Contentons nous ici d'énoncer les questions. Les relations entre le tout de l'organisme et de ses parties, entre le fonctionnement cellulaire et l'organisme dans son milieu. Les relations entre le psychique et le somatique. L'étude des maladies pour elles-mêmes et non comme simple faire-valoir de la biologie. L'étude des maladies comme exploration anthropologique des normes physiologiques de fonctionnement. L'analyse du cerveau et de la pensée à partir de modèles chimiques ou du modèle de l'ordinateur. L'analyse réductrice du langage et de la mémoire. Toutes ces difficultés se retrouvent dans la lente constitution d'une biologie théorique et d'une médecine théorique. On peut également en trouver les éléments constitutifs dans les procédés tronqués de la vulgarisation scientifique, ou l'usage publicitaire de la science.

En un mot, il n'y a pas de critère a priori de scientificité, ce qui ne signifie pas que l'on ne puisse distinguer, a posteriori l'activité scientifique et ses produits, des autres activités humaines. L'activité scientifique est un processus qui invente des rationalités historiques successives. Elle inclut sa propre rectification. La valeur réellement éducative et culturelle d'un enseignement scientifique, donc sa valeur émancipatrice, est à ce prix.

\section{RÉSUMÉS}

Peut-on s'interroger sur la spécificité de l'enseignement de la biologie sans être accusé de corporatisme défensif? Doit-on inscrire cette question dans le cadre d'un enseignement scientifique, ou dans une interdisciplinarité encore plus audacieuse? Rechercher des frontières ne consiste pas nécessairement à construire les murs d'une forteresse. Une frontière est un point de passage et donc un lieu d'échange et d'ouverture contrôlé. Tout dépend de la nature du contrôle. Ce texte prétend rouvrir les frontières sans les effacer.

Can one possibly put into doubt the specific nature of the teaching of biology without being accused of having a reactionary attitude. Should this issue be put within the scope of science instruction, or else within that of an even more daring interdisciplinarity? This text's intent is to reopen the boundaries between fields of knowledge without doing away with them.

\section{INDEX}

Mots-clés : anti-intellectualisme, frontière du savoir, réductionnisme

Keywords : anti-intellectualism, field of knowledge boundary, reductionism 
AUTEUR

GUY RUMELHARD

Institut National de Recherche Pédagogique, lycée Condorcet, Paris 